Review

\title{
Small Molecule Sequential Dual-Targeting Theragnostic Strategy (SMSDTTS): from Preclinical Experiments to- wards Possible Clinical Anticancer Applications
}

\author{
Junjie Li1 ${ }^{1,2}$, Raymond Oyen ${ }^{1}$, Alfons Verbruggen ${ }^{3}$, Yicheng $\mathrm{Ni}^{1,2, \mathbb{}}$ \\ 1. Department of Imaging and Pathology, Biomedical Sciences Group; KU Leuven, Belgium. \\ 2. Molecular Small Animal Imaging Center, Faculty of Medicine; KU Leuven, Belgium. \\ 3. Laboratory of Radiopharmacy, Faculty of Pharmaceutical Sciences, KU Leuven, Belgium.
}

$\triangle$ Corresponding author: Prof. Yicheng Ni, Theragnostic Laboratory, Department of Imaging \& Pathology, Biomedical Sciences Group, KU Leuven, Herestraat 49, BE-3000 Leuven, Belgium. Tel: +32-16-33.01.65, Fax: +32-16-34.37.65. E-mail: yicheng.ni@med.kuleuven.be.

(c) Ivyspring International Publisher. This is an open-access article distributed under the terms of the Creative Commons License (http://creativecommons.org/ licenses/by-nc-nd/3.0/). Reproduction is permitted for personal, noncommercial use, provided that the article is in whole, unmodified, and properly cited.

Received: 2012.11.30; Accepted: 2013.01.03; Published: 2013.01.22

\begin{abstract}
Hitting the evasive tumor cells proves challenging in targeted cancer therapies. A general and unconventional anticancer approach namely small molecule sequential dual-targeting theragnostic strategy (SMSDTTS) has recently been introduced with the aims to target and debulk the tumor mass, wipe out the residual tumor cells, and meanwhile enable cancer detectability. This dual targeting approach works in two steps for systemic delivery of two naturally derived drugs. First, an anti-tubulin vascular disrupting agent, e.g., combretastatin A4 phosphate (CA4P), is injected to selectively cut off tumor blood supply and to cause massive necrosis, which nevertheless always leaves peripheral tumor residues. Secondly, a necrosis-avid radiopharmaceutical, namely ${ }^{131} \mid$-hypericin $\left({ }^{131} \mid-H y p\right)$, is administered the next day, which accumulates in intratumoral necrosis and irradiates the residual cancer cells with beta particles. Theoretically, this complementary targeted approach may biologically and radioactively ablate solid tumors and reduce the risk of local recurrence, remote metastases, and thus cancer mortality. Meanwhile, the emitted gamma rays facilitate radio-scintigraphy to detect tumors and follow up the therapy, hence a simultaneous theragnostic approach. SMSDTTS has now shown promise from multicenter animal experiments and may demonstrate unique anticancer efficacy in upcoming preliminary clinical trials. In this short review article, information about the two involved agents, the rationale of SMSDTTS, its preclinical antitumor efficacy, multifocal targetability, simultaneous theragnostic property, and toxicities of the dose regimens are summarized. Meanwhile, possible drawbacks, practical challenges and future improvement with SMSDTTS are discussed, which hopefully may help to push forward this strategy from preclinical experiments towards possible clinical applications.
\end{abstract}

Key words: small molecule sequential dual-targeting theragnostic strategy; SMSDTTS; vascular disrupting agent; combretastatin A4 phosphate; necrosis avid contrast agent; hypericin.

\section{Introduction}

Conventional anticancer treatments such as cytotoxic chemotherapy and radiotherapy are limited by serious side effects arising from their intrinsic toxicities, and are generally far from curative (1). Though recent advances have been achieved with targeted molecular anticancer therapies, inherent hurdles for cancer cure through nonexclusive mechanisms cannot be ignored. For instance, target mutation and escape, target amplification, and down/upregulation of mechanisms may lower the intracellular drug con- 
centration and/or activation of complementary pathways $(2,3)$. On the other hand, cancer stem cells (CSCs) are located in the specific microenvironment and are generally resistant to chemotherapies; these surviving CSCs then repopulate the tumor, causing relapse. Thus, hitting the evasive targets in cancer still proves a major challenge $(4,5)$. Clinically, tumor residue after therapies presents always a culprit for cancer recurrence and patient death.

In developing highly efficient targeting cancer therapies, there are two additional opportunities. First, homeostatic factors that compose up to $90 \%$ of the tumor mass in some tumors have great essentiality to influence the level of malignant aggression and treatment outcomes (6). Homeostatic factors render tumor microenvironment also as a therapeutic target besides cancer cells (7). Secondly, necrotic tissues, which comprise $30-80 \%$ of a solid tumor and locate always close to the viable cancer cells, can function as a universal anchor for targeting malignancies (8-11). Therapeutic use of iodine-131 in patients with thyroid cancer has been proven highly effective with ablation rate over $80 \%(12,13)$. In order to translate such a success to broader indications or even a generalized strategy for solid tumors, efforts have been made to render tumor affinity and sensitivity to iodine-131, i.e., allowing iodine-131 to highly concentrate inside viable tumor cells or close to them within the beta-irradiation range. That way, improved treatability or even curability can be expected for patients with different kinds of solid tumors. Furthermore, various other therapies of synergistic or complementary antitumor effects with different mechanisms can be combined for more thorough elimination of cancer cells.

Recently, a general and unconventional anticancer approach namely small molecule sequential dual targeting theragnostic strategy (SMSDTTS) has been introduced (11). SMSDTTS aims to target and debulk the tumor mass, wipe out the residual tumor cells, and meanwhile enable cancer detectability. SMSDTTS works in two-steps for systemic delivery of two naturally derived drugs. First, an anti-tubulin vascular disrupting agent (VDA) such as combretastatin A4 phosphate (CA4P) is injected to selectively cut off tumor blood supply and to cause massive necrosis, which though always leaves peripheral residues. Secondly, a necrosis-avid radiopharmaceutical such as ${ }^{131} \mathrm{I}-\mathrm{Hyp}$ ericin (131 I-Hyp) is administered the next day, which accumulates in intratumoral necrosis and irradiates the neighboring cancer cells with beta particles to prevent tumor relapse. Theoretically, this complementary targeting approach may biologically and radioactively ablate solid tumors and reduce the risk of local recurrence, remote metastases, and thus cancer mortality. Meanwhile, the gamma rays emitted by iodine-131 enable radio-scintigraphy to detect the tumors and follow up the therapy, hence constituting a simultaneous theragnostic approach (Fig. 1). Based on the above-mentioned unique rationales, SMSDTTS may precisely hit two stable targets within the stroma domain by selectively destroying the tumoral vessels and sterilizing tumor microenvironment. Thus, the SMSDTTS seems advantageous over other existing therapies and deserves further preclinical and clinical development (11).

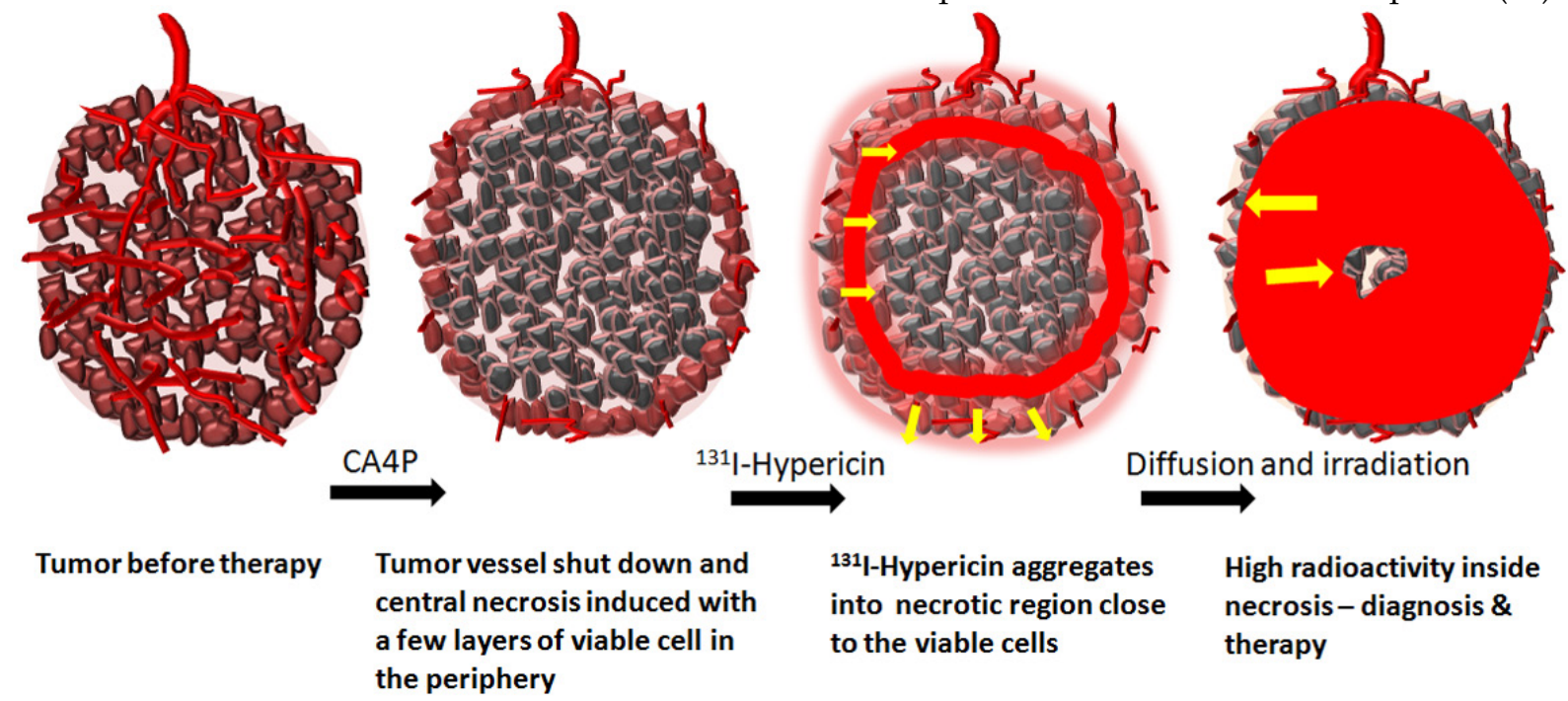

Figure I. Schema illustrates the mechanisms of the small molecule sequential dual-targeting anticancer strategy (SMSDTTS): first to treat the solid tumor and to cause massive tumor necrosis by using CA4P. After being iv injected 24h later, 131I-Hyp accumulates in the intratumoural necrosis bordering the viable rim and gradually diffuses into the necrotic center. Diagnostic and therapeutic efficacies are achieved owning to the constant gamma and beta radiation. 
Exploiting the true natural power, this new strategy has now shown promise in multicenter animal experiments, and may demonstrate superior anticancer efficacy and clinical safety in upcoming preliminary clinical trials. In this short review article, information about the two involved agents, the hypotheses/rationales, preclinical antitumor efficacy, multifocal targetability, and simultaneous theragnostic property of SMSDTTS in different animal tumor models, as well as toxicities of the dose regimens are summarized. Meanwhile, possible drawbacks, practical challenges and future improvement with SMSDTTS are also discussed, which hopefully may help to push forward this strategy from preclinical experiments towards possible clinical applications.

\section{Tumoral vasculature and VDAs}

Tumor vasculature often features abnormal morphology and functionality such as a lack of pericytes or deficiency of pericyte function, irregular diameters, and thin, tortuous, and leaky walls $(14,15)$. Meanwhile, distinct molecular markers are found in the pericytes, endothelium, and extracellular matrix of tumor vessels. These specializations promote the tumors to form their own vessels that function as channels for nutrition and metabolite exchange within the tumor (15). Such distinct features of tumor vessels have been exploited as specific targets for drug discovery and cancer therapies (16).

VDAs target either the endothelial cells lining tumor vessels or proteins derived from the tumor endothelium (17-19). The effects of VDAs are inducing direct damage to the pre-existing tumoral endothelium, and causing collapse of the vasculature inside solid tumors. Therefore, the tumor is deprived of oxygen supply or prohibited from blood flow, which consequently leads to rapid hemorrhagic necrosis or tumor cell death (3). There are two categories of VDAs under investigation, i.e. ligand-directed biologic VDAs and small molecular VDAs (20). Ligand-directed VDAs are antibodies, peptide growth factors and toxic proteins that deliver toxins, procoagulant, and pro-apoptotic effectors to the receptors expressed on the surface of endothelial cells in tumoral vessels (21). Small molecular VDAs selectively target tumor blood vessels through either induction of local cytokine production or depolymerization of tubulin in tumoral endothelia (20-22). Small molecular VDAs are further designated into two classes: synthetic flavonoids and tubulin-binding agents. Synthetic flavonoids work through tubulin-independent mechanisms by direct disruption of tumor vascular endothelial cells via apoptosis induction, or by indirect upregulation of cytokines including tumor necrosis factor-alpha, interferon and interleukins (23-26). Tubulin-binding agents bind to either the colchicine or vinblastine binding sites on tubulin, rapidly induce depolymerization of microtubules in endothelial cytoskeletons, and then increase vascular permeability. Thereafter, obstruction of tumor blood flow causes hypoxia and eventually leads to tumor necrosis (27-29). However, exceptions do exist. For example, some tumor vessels are derived from pre-existing normal vessels that are insusceptible to VDAs and peripheral tumor tissues can also obtain their nutrients from the surrounding normal tissues by diffusion. Therefore, independent of the types of VDA used, the results of VDA therapies to the tumor are the same, i.e. collapse of tumor vasculature and massive tumor necrosis but accompanied with a shell of viable tumor cells (Fig. 2) (30-32).

\section{CA4P as one of the most potent VDAs}

Among a number of VDAs that are being pursued, CA4P (MW= $442 \mathrm{Da})$ is a leading small molecular tubulin-binding drug that is at the forefront in research and clinical settings $(33,34)$. CA4P is the water-soluble phosphate prodrug of combretastatin A4 (CA4), which is extracted from South African willow tree Combretum caffrum $(21,35)$. Following intracellular uptake, CA4P is in vivo dephosphorylated by non-specific endogenous phosphatases into the active form of CA4. The latter then binds to the colchicines-binding site in beta-tubulin of microtubules (36). Although structurally similar, the binding of CA4 is more rapid and reversible with greater affinity compared with that of colchicines $(27,36)$. An immediate vascular collapse can be induced following injection of CA4P at a dose of one-tenth the maximum tolerated dose (MTD) in tumor models (22). CA4P can cause extensive central necrosis up to $90 \%$ of tumor mass $(37,38)$ with a few layers of viable cells left as a thin rim of peripheral tumor (Fig. 2). In preclinical models, the remaining viable tissues are always attributed to rapid tumor regrowth following single-agent administration (39). Therefore, treatments with CA4P were frequently combined with a variety of cytotoxic agents, radiotherapy and antiangiogenesis inhibitors, with the intention to achieve enhanced tumoricidal effects (40-42). Despite the challenges of viable tumor residues left behind, the massive tumor necrosis caused by CA4P or other VDAs does provide new opportunities for further targeting therapy as addressed by SMSDTTS. 


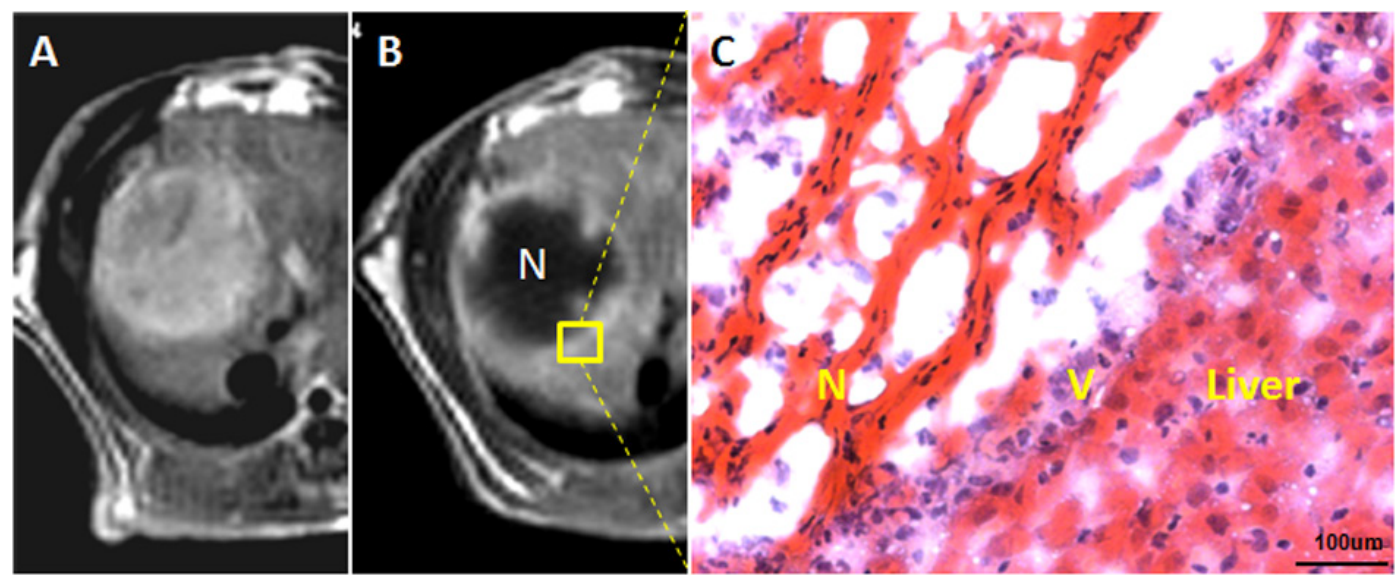

Figure 2. Vascular shutdown effect and subsequent intratumoral necrosis caused by iv injection of CA4P: A. hepatic Rhabdomyosarcoma-I (RI) tumor in rat was shown on contrast enhanced TI-weighted MR image; B. minutes to hours after CA4P injection, a hypointense tumor is surrounded by a hyperenhanced rim, suggesting vessel shutdown and tumor necrosis with peripheral viable residues; C. the corresponding histological section proves massive intratumoral necrosis $(\mathrm{N})$ and a few layers of viable tumor cells $(\mathrm{V})$ in the periphery adjacent to the normal liver.

\section{Necrosis and necrosis avid contrast agents (NACAs)}

Instead of programmed cell death or apoptosis as a result of a well orchestrated signaling cascade, necrosis has classically been characterized as the consequence of a passive, degenerative, and accidental or uncontrolled form of cell death resulting mainly from environmental perturbations. Intoxication, infection, trauma, and any other factors that can attack the cellular ability to complete mitosis, to maintain cellular membrane integrity, and to control release of inflammatory contents are often causative to the occurrence of necrosis $(43,44)$. Necrotic tissue is a principal feature of numerous pathologies and diseases such as hypoxia, ischemia, hypoglycaemia, poisoning, sepsis, organ infarction and dysfunction, atherosclerosis, pancreatitis, stroke, drug induced cell or organ toxicity and malignancies, etc. $(45,46)$.

Imaging of necrotic tissue has the potential to detect necrosis related disorders. Porphyrins and derivatives have initially been investigated as tumor-localizing agents for cancer photodynamic therapy $(47,48)$. Porphyrin analogues have also been exploited for developing paramagnetic metalloporphyrins as "tumor-seeking" contrast agents for magnetic resonance imaging (MRI). In the early 1990s, the previously reported "tumor specificity" of prophyrin analogues was redefined to be "necrosis avidity" (47-49). Therefore, the term "necrosis avid contrast agents (NACAs)" was proposed by $\mathrm{Ni}$ et al and NACAs have become one of the focused research interests over the past two decades $(47,50)$. They described the necrosis avidity of NACAs in a series of experiments using MRI-microangiography/ fluorescence-histology colocalization techniques (47-53). Although the underlying mechanisms still need to be elucidated, the following phenomena of necrosis-avidity have been extrapolated. After systemic administration, NACAs enter the necrotic area by the combined effects of blood perfusion, drug extravasation, and interstitial diffusion. Certain intracellular radicle expose during necrosis process and physicochemically interact with a variety of endogenous and exogenous necrosis avid chemicals to form strong bonds and these chemicals can be utilized as NACAs (53). Using certain imaging modalities, NACAs may enable in vivo visualization and quantification of necrosis or infarction for diagnostic purposes to determine the severity, estimate prognosis and monitor therapeutic response of the disease; meanwhile NACAs can also be exploited to formulate new therapeutic strategies (54) as exemplified by the introduction of SMSDTTS.

\section{NACAs in MRI and Nuclear Imaging}

Efforts have been made to improve the localization of necrosis and infarction by using NACAs in noninvasive imaging. Much attention has been paid to "necrosis-specific" diagnosis to discriminate between viable and nonviable myocardium with contrast enhanced MRI. Traditional contrast agents such as Gd-DTPA (Gadopentetic acid) are incapable of making explicit distinction between viable and necrotic tissues due to their inherent nonspecific nature and related imaging interpretations are inaccurate, uncertain, and dependent on multiple influential factors (47). Over the past decades, a series of porphyrin and 
nonporphyrin

NACAs

Mn-tetraphenylporphyrin

(Mn-TPP)

including

bis-Gd-DTPA-mesoporphyrin (Gd-MP, or Gadophrin-2), bis-Gd-DTPA-pamoic acid (ECIII-60), and bis-Gd-DTPA-benzylidene-bis (indole-2-acetic acid hydrazide) (ECIV-7) have been introduced and have exhibited high necrosis avidity and diagnostic capacity in differential diagnoses between reversible ischemic injury and irreversible infarct, acute and chronic myocardial infarction, and occlusive and reperfused myocardial infarction in animal experiments using MRI $(52,53)$.

Focused interests in diagnosis of necrosis also cover nuclear scintigraphic imaging. 99mTc-pyrophosphate, $111 \mathrm{In} / 99 \mathrm{~m}$ Tc-antimyosin monoclonal antibody (mAb) Fab, and $99 \mathrm{mTc}$-glucarate are some of the NACAs that have shown diagnostic potentials either in animal experiments or in clinical uses. ${ }^{99 m T c-p y r o p h o s p h a t e ~ i s ~ s u p p o s e d ~ t o ~ b i n d ~ t o ~ n e-~}$ crotic myocardium by targeting calcium phosphate deposited in the mitochondria of infarcted or severely injured myocardium (56,57). 111In/99mTc-antimyosin $\mathrm{mAb} \mathrm{Fab}$ is a monoclonal antibody fragment specific

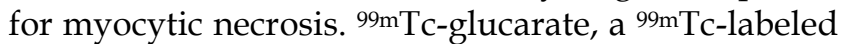
simple dicarboxylic acid, is supposed to bind to positively charged histones in disintegrated nuclei and reduced sub-cellular organelle proteins in necrotic myocytes (58). Although promising, each of these NACAs has its own limitations. e.g., due to the insufficient diagnostic accuracy and limited stability of 99mTc-pyrophosphate, it has never gained widespread use $(50,58)$. Clinical indications of ${ }^{111}$ In-antimyosin $\mathrm{mAb}$ were restrained only to ischemic heart disease due to its high but narrow specificity, unaffordable costs, and possible immunogenic side effects $(50,55,58)$. ${ }^{99 m T c}$-glucarate only works in the early hours after the onset of acute injury (59).

\section{Exceptional necrosis affinity of hypericin (Hyp)}

Besides the above mentioned NACAs, a large variety of synthetic or natural substances that share a common necrosis-avidity were exploited as potent NACAs despite their diverse chemical structures.

Hyp, chemically, 4,5,7,4',5',7'-hexahydroxy-2,2'dimethylnaphthodianthrone (MW=504 Da), is a polyphenolic polycyclic quinone originally found in St. John' s wort (Hypericum perforatum). Hyp exhibits a wide range of pharmacologically interesting properties. Remarkable characteristics and clinical applications of this compound include its antidepressant, antiviral, and antitumoral properties, etc. (60). Being a powerful naturally occurring photosensitiser, Hyp is applied in photodynamic therapy (PDT) for treatment of cancer in animal experiments $(61,62)$. The potent photosensitizing property makes Hyp possibly the most powerful naturally occurring photosensitizer so far described (63). Various studies have suggested specific molecular interactions that account for the cellular localization or tumoritropic proterty of Hyp (64-67). Despite numerous pharmacological findings published concerning the potential clinical use of Hyp, the preclinical efficacy for the above applications still remains controversial (60). Nevertheless, in recent research on contrast agents, $\mathrm{Ni}$ et al. further expanded the role of Hyp in medicine by revealing its necrosis avidity superior to its tumor affinity $(47,58)$. The exceptional necrosis avidity of Hyp has been proven in vivo by the highest necrosis-to-viable tissue ratios among ever discovered specific agents $(47,58,68)$. Therefore, radiolabeled derivatives of Hyp, e.g., ${ }^{123} \mathrm{I}-\mathrm{Hyp},{ }^{64} \mathrm{Cu}$-bis-DOTA-Hyp, etc., have been designed for diagnosis of various diseases utilizing their necrosis avidity $(58,68-73)$.

\section{Necrosis targeted therapy to malignancies}

The introduction of NACAs constitutes a potential strategy not only for detecting various diseases, but also for developing therapeutic agents to target malignancies. Often the absorbed dose to the lesions or tumors has been too low owing to the unfavorable pharmacokinetics of the therapeutic agents (74). A high specificity guarantees a high dose of drug on targeted tissues and minimizes toxicity on normal cells. Tumor necrosis therapy (TNT) was designed using a genetically engineered, iodine-131 labeled chimeric $\mathrm{mAb}$, which is specific for a universal nuclear antigen histone present only on dead or dying cells $(8,75)$. However, the poor pharmacokinetic profile, low tumor uptake owing to their large molecular mass, insufficient density of target antigens on tumor spots, as well as short serum half-life, have limited its clinical use (76-78).

Following recent advances, Hyp labeled with iodine-131 (131-Hyp) was proposed for the complementary treatment of remnant cells with prior induced massive tumoral necrosis using CA4P $(3,11,76,79)$. Compared with peptide based or antibody mediated agents, which usually show fast washout from tumor $(<72 \mathrm{~h})$, the extraordinary necrosis avidity as well as prolonged retention of 131I-Hyp within targeted tissues ( $>30$ days) assured an effective treatability, targetability and detectability of tumors that contain large necrotic components (Fig. 3) $(11,76,80-82)$. 

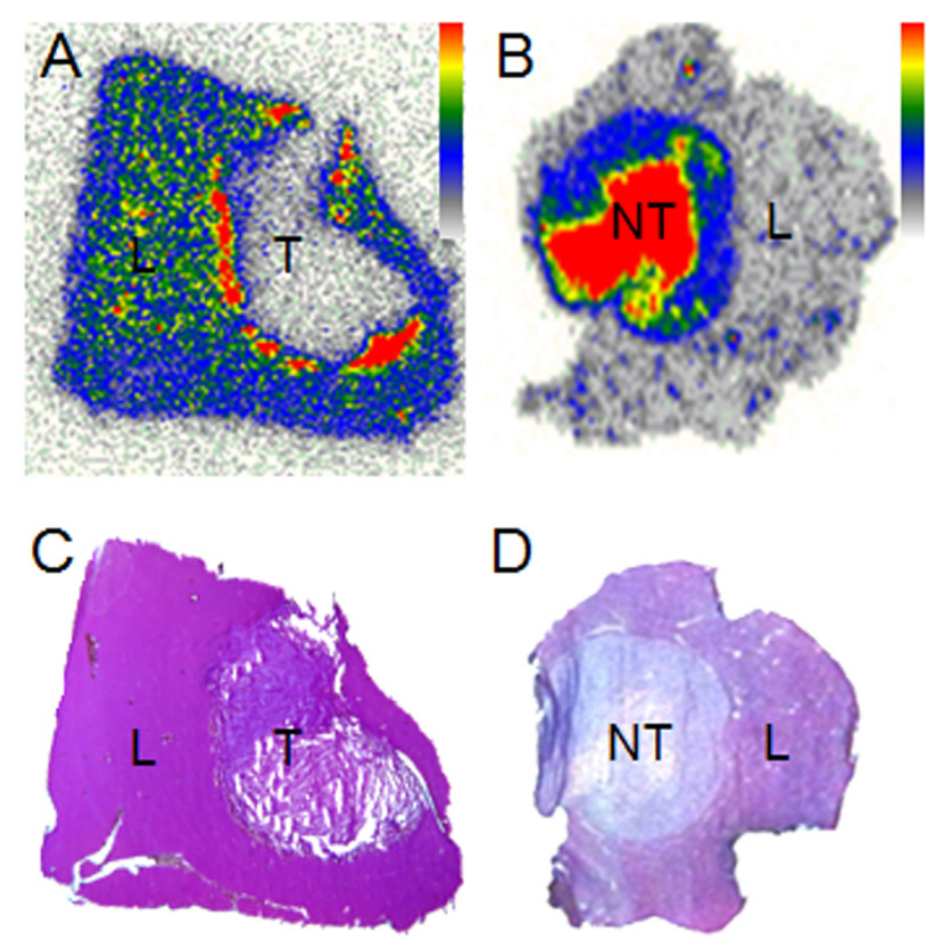

Figure 3. Autoradiographic and histologic findings: A. within a few hours to 2 days after injection of radioiodinated Hyp in CA4P pretreated hepatic RI tumor in rats, autoradiogram reveals a rim of high radioactivity in the necrosis bordering the peripheral viable cells; $\mathrm{B}$. after a few days, high radioactivity mainly accumulates in the necrotic core, as verified with the corresponding histological sections of $C$ and D, respectively (L: liver; T: tumor; NT: necrotic tumor).

\section{Engineered designs with SMSDTTS}

The concept of SMSDTTS has been gradually formulated during the last 20 years of research on NACAs and VDAs with a soil-to-seeds hypothesis (11). Combining multiple agents or strategies for targeting different molecular pathways can elevate the genetic barriers for cancer cell mutations. Multiple drugs with cross-talk mechanisms could generate synergistic effects with higher targeted selectivity and better clinical outcomes $(83,84)$. Following CA4P injection, the endothelium of tumoral capillary vessels is selectively targeted, which subsequently causes collapse and shutdown of tumor vasculature. Thereafter, tumor cells are deprived from major blood and oxygen supply, and hemorrhagic necrosis happens inside the tumor within minutes to hours after injection $(14,17,85,86)$. However, few layers of viable tumor cells at the periphery always survive CA4P treatment, and eventually lead to tumor relapse. With the aim to complement CA4P treatment, ${ }^{131} \mathrm{I}-\mathrm{Hyp}$ is intravenously administrated 24 hours after the CA4P dose to eliminate the remaining cancer cells and to prevent tumor regrowth (Fig. 1,3,4) $(42,87,88)$. The engineered design for SMSDTTS that combines sequential intra- venous injection of CA4P and 131 I-Hyp at a $24 \mathrm{~h}$ interval is believed advantageous for the following considerations: a) being both naturally derived small molecules allowing systemic injections with much better accessibility to targeted tissues, CA4P and Hyp (in the form of its radiolabeled iodo-derivative) share a highly targeting capacity but diverse and complementary specificities; b) massive intra-tumoral necrosis induced by CA4P serves as a target or anchor for ${ }^{131}$ I-Hyp; c) a $24 \mathrm{~h}$ interval between CA4P and ${ }^{131} \mathrm{I}-\mathrm{Hyp}$ allows the complete formation of necrosis, meanwhile optimal accessibility to the latter due to partial recovery of tumor vascularization (89); d) iodine-131 that emits tumoricidal beta particles and gamma rays useful for scintigraphy provides a theragnostic solution for malignancies; e) being spatially or geographically cooperational, CA4P kills tumor cells inside out, whereas ${ }^{131}$ I-Hyp eradicates the remnant tumor cells on the periphery. Micro-geographically, ${ }^{131}$ I-Hyp accumulates with high concentration into the necrotic tissue neighboring the viable cancer cells. A few layers of remaining viable cells in the periphery are generally within the 2-mm irradiation range of the beta particles emitted from iodine-131 (90); f) anticancer effects of the two compounds are synergistic, since the 
peripheral remnant tumor cells following the injection of CA4P are generally oxygen-saturated and rapid proliferating cells that are more sensitive to radiation. Meanwhile, the constant crossfire radiation from the energetic beta particles of iodine-131 could eradicate all cells within the beta particle penetration range, enabling greater synergistic therapeutic efficacy; g) the radiating stroma and harsh microenvironment are no longer suitable for either re-growth of tumor cells or normalization of tumoral vasculature $(17,91)$; and h) reduced cytotoxicity to the surrounding normal tissues as a result of the different mechanisms of the two drugs as well as the time interval between the two administrations.

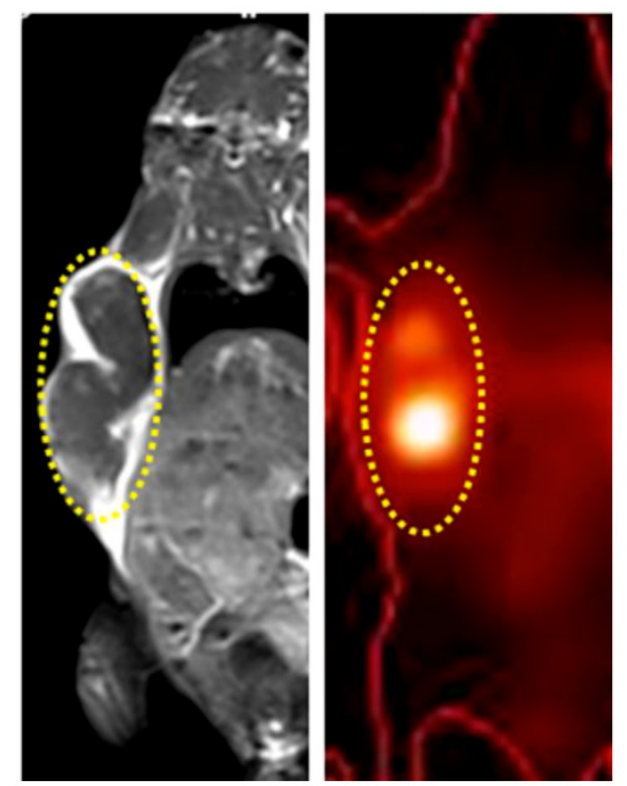

Figure 4. Diagnostic capacity of SMSDTTS is shown in a mouse model bearing subcutaneous tumors. Dual-focal hot spots in colocalized planar gamma scintigraphy/CT image (right) correspond exactly to the hypointense tumor core on the MR image (left) 12 days after treatment.

\section{Current progresses with research on SMSDTTS}

In agreement with the EU regulations for the research and development of radiopharmaceuticals (92), in vivo studies using radiolabeled derivatives of Hyp and SMSDTTS have been conducted using different animal models with diverse tumors and necroses $(11,58,69-73,79,90)$. Related toxicity studies have also been performed $(93,94,95)$.

\section{Radioloabeling of Hyp}

The radioloabeling of Hyp with radioiodine (123I for in vivo diagnosis, ${ }^{125}$ I for in vitro studies, ${ }^{131}$ I for radiotherapy in combination with gamma scintigra- phy) are continuously optimized to enable higher labeling yields, better pharmacokinetics and in vivo stability. With a standard electrophilic radioiodination method in the presence of peracetic acid, an overall labeling yield of $75-80 \%$ was achieved in the radiolabelling of mono-[123I]iodohypericin monocarboxylic acid and mono-[123I]iodohypericin $(69,70,96)$. More recently, the iodogen coating method was used for radio-iodination of Hyp, resulting in a labeling efficiency above $99.5 \%$ (90). Further optimization in labeling and formulation is being studied.

\section{Biodistribution of radioiodinated Hyp derivatives}

Biodistribution and necrosis avidity of radioiodinated Hyp have been explored in different animal models of pathologies $(11,58,69,70,90,96,97)$. Biodistribution studies of ${ }^{123}$ I-Hyp in mice showed that the tracer is mainly cleared via the hepatobiliary pathway, which results in high percentages of over $70 \%$ radioactivity excreted via the intestines and feces, and about $10 \%$ of the injected dose excreted in the urine at $24 \mathrm{~h}(70,97)$. At $60 \mathrm{~h}$ after injection in rats, a high concentration of ${ }^{123} \mathrm{I}-\mathrm{Hyp}$ (3.51 percent injected dose per gram of tissue, \% ID/g) in the reperfused necrotic part of the liver, and $0.38 \% \mathrm{ID} / \mathrm{g}$ in the normal liver was observed (58). In a rat model of liver rhabdomyosarcoma-1(R1), 131I-Hyp was found to accumulate in tumor necrotic regions at $3.13 \% \mathrm{ID} / \mathrm{g}$, corresponding to a target-to-liver activity ratio over 20 on day 8 after injection (11). In mice carrying subcutaneous radiation-induced fibrosarcoma (RIF-1), high concentration of ${ }^{131} \mathrm{I}-\mathrm{Hyp}$ remained in tumor necrotic regions over 30 days, indicating a good and long term in vivo stability $(11,82)$. Micro-geographically, the autoradiography of tumor slices in combination with analysis of the corresponding histological sections revealed that a high concentration of radioiodinated Hyp first accumulated into the edge of necrosis bordering the peripheral viable cells, and the radiotracer gradually diffused into the necrotic core of the tumor (Fig. 1, Fig. $3)$.

\section{Diagnostic potential of radioiodinated Hyp or SMSDTTS}

Sufficient imaging resolution for necrotic lesions in different animal models was achieved with nuclear gamma scintigraphy after administration of ${ }^{123} \mathrm{I}-\mathrm{Hyp}$ (97). At $24 \mathrm{~h}$ and $48 \mathrm{~h}$ after ${ }^{123}$ I-Hyp injection, well defined hot spot imaging was persistently visualized in vivo with single photon emission computed tomography (SPECT) in rats with reperfused hepatic infarction and rabbit models of myocardial infarction (58). In rabbit models of acute occlusive myocardial 
infarction, $\mu$ SPECT images obtained at $9 \mathrm{~h}$ post injection revealed a good match between perfusion defect and area of high ${ }^{123}$ I-Hyp uptake, suggesting the high sensitivity of ${ }^{123} \mathrm{I}-\mathrm{Hyp}$ for detecting small infarcts (70). In a rat model of hepatic R1, hot spot imaging using planar gamma scintigraphy in combination with CT colocalised tumor necrosis at $24 \mathrm{~h}$ post ${ }^{131} \mathrm{I}-\mathrm{Hyp}$ injection $(11,90)$. In a murine model with subcutaneous xenografts of RIF-1, ${ }^{131}$ I-Hyp allowed imaging of tumor necrosis using planar gamma scintigraphy/CT from $24 \mathrm{~h}$ till over 12 days after injection, with $100 \%$ sensitivity for tumor detection (Fig. 4) (82).

\section{Tumoricidal effects}

Tumoricidal effects were successively revealed with SMSDTTS and ${ }^{131}$ I-Hyp solely. In nude mice bearing subcutaneous RIF-1, stabilization in tumor growth for 5 days was reported after three injections of only ${ }^{131}$ I-Hyp over a 3-week treatment period (79). In a rat model of liver R1, tumor size at 8 days after treatment was significantly smaller in a SMSDTTS treated group compared with the vehicle-control group as well as the single-targeting groups that were treated with CA4P or ${ }^{131}$ I-Hyp solely $(\mathrm{p}<0.01)$ within 8 days $(11,98)$. More recently, prolonged survival was found $(p<0.01)$ in a murine model of subcutaneous RIF-1 in SMSDTTS treated group compared with the vehicle control and single CA4P groups, with a median survival 33, 22, and 21 days, and, at day 15, the corresponding tumor doubling times of $7.8 \pm 2.8,4.5 \pm$ 0.5 , and $4.4 \pm 0.67$ days, respectively (82).

\section{Toxicity concerns regarding iodinated Hyp}

The radio-toxicity of radioiodine- 131 has been well defined in various clinical applications during the past few decades (99-101). In recent toxicity study with non-radioactive mono-iodohypericin (I-Hyp), all mice tolerated well iv injections of 0.1 and $10 \mathrm{mg} / \mathrm{kg}$ without any signs of clinical toxicity or detectable side effects. Clinical blood biochemistry tests did not reveal any significant differences between normal control and I-Hyp treated mice. The half lethal dose $\left(L_{50}\right)$ of I-Hyp is above 180 times of the experimental dose used in SMSDTTS, suggesting a negligible chemotoxicity with radioiodinated Hyp (95). Good safety profiles were also demonstrated with the reagents such as full dimethyl sulfoxide (DMSO) and full Iodogen involved in the pharmaceutical preparation of SMSDTTS $(94,95)$. Altogether these data suggest a strong evidence for the controllable and acceptable safety of SMSDTTS for its possible future clinical applications in terms of both radio- and chemotoxicity.

\section{Possible drawbacks, practical challenges and future improvement with SMSDTTS}

Each of the two drugs involved in SMSDTTS has its own defects for clinical applications. CA4P is still under phase I-III clinical trials on different indications. Clinical investigations with CA4P mainly focus on hypervascular malignancies including ovarian, lung, and anaplastic thyroid cancers. Although generally well tolerated, dose limiting toxicities of CA4P mainly include cardiotoxicity and/or chest pain, reversible ataxia, vasovagal syncope, and motor neuropathy $(35,102,103)$. There are still unmet needs for $\mathrm{CA} 4 \mathrm{P}$ with more definite indications and favorable safety profiles.
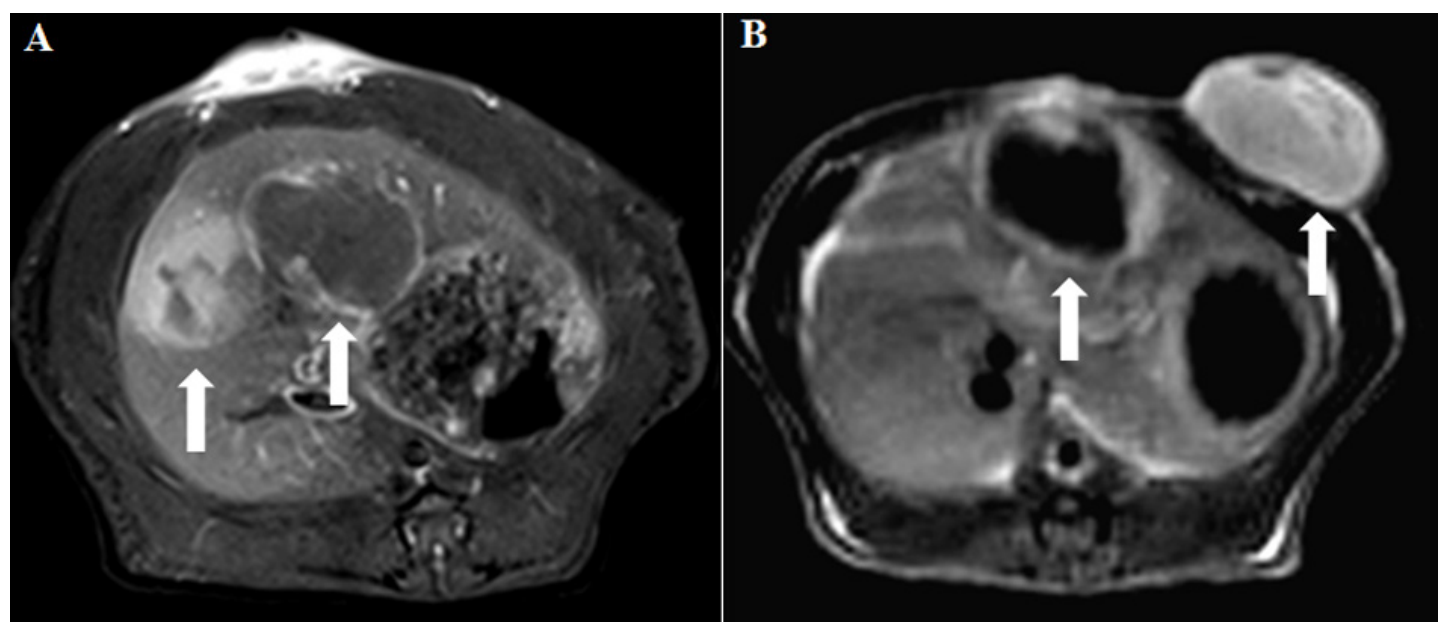

Figure 5. Inhomogeneity of vessel shutdown induced by CA4P is shown with CE-MRI in rat models of engrafted bifocal rhabdomyosarcoma-I (RI): A. white arrows indicate the different extents of vessel shutdown between hepatic tumors; and B. between hepatic and subcutaneous tumors. 
Imaging of necrosis with ${ }^{123}$ I-Hyp using in vivo planar gamma scintigraphy or SPECT is limited by the relative short physical half-life of $13 \mathrm{~h}$ for iodine-123. Due to the considerable systemic distribution of radioactivity at an early stage after administration of ${ }^{123} \mathrm{I}-\mathrm{Hyp}$, sufficient imaging resolution for necrotic lesions cannot be easily achieved within $9 \mathrm{~h}$ $(58,70)$. For tumoral imaging, it is difficult to definitely contour the tumor within $24 \mathrm{~h}$ after injection because of the relative insufficient spatial resolution of planar gamma scintigraphy and the no-entry zone caused by the vascular shutdown effect of CA4P (90). Although iodine-131 with a physical half-life over 8 days allows long term imaging of necrosis, ${ }^{131}$ I-Hyp is not suitable for use in certain patients such as those with acute myocardial infarction because of the possible cytotoxicity to healthy heart tissues. For diagnostic purposes, iodine-124 as a relatively long-lived positron emitter $(\mathrm{T} 1 / 2=4.18$ days $)$ can be a better substitute for iodine-123. Advantages with ${ }^{124}$ I-Hyp include: a) the possibility of reliable and over time quantitative analysis of tracer kinetics, b) allowing up to 10 days post injection for imaging; c) allowing 4D microPET/CT scanning and quantification; and d) better imaging resolution of PET in comparison to SPECT.

Table I. Possible substitutes and complements for the two therapeutic components involved in the current SMSDTTS and their future improvements.

\begin{tabular}{|c|c|}
\hline Creation of tumoral necrosis & $\begin{array}{c}\text { Targeting necrosis and eradiating remaining } \\
\text { viable cells }\end{array}$ \\
\hline \multicolumn{2}{|l|}{ CA4P } \\
\hline \multicolumn{2}{|c|}{ Possible substitutes/complements for CA4P and ${ }^{131} \mathrm{I}$-hypericin } \\
\hline $\begin{array}{l}\text { - } \text { Surgery } \\
\text { - } \text { Chemotherapy } \\
\text { - Other VDAs } \\
\text { - Radiotherapy } \\
\text { - Radiofrequency ablation (RFA) } \\
\text { - Targeted cancer therapies, e.g. SMKIs, } \\
\text { mAbs, etc. }\end{array}$ & $\begin{array}{l}\text { - }{ }^{131} \text { I-chTNT-1/B mAb (TNT) } \\
\text { Other necrosis avid agents labeled with } \\
\text { beta-minus particle emitting radioisotope, } \\
\text { e.g., compounds derived from porphyrins, } \\
\text { pamoate, benzylidene bis-indole, } \\
\text { pyrophosphate, antimyosin mAb-Fab, } \\
\text { glucarate, etc. }\end{array}$ \\
\hline \multicolumn{2}{|l|}{ Possible approaches to optimize the SMSDTTS } \\
\hline $\begin{array}{l}\text { - Multi-cycle treatments } \\
\text { - Single dose of CA4P + repeated doses } \\
\text { of }{ }^{131} \text { I-Hyp } \\
\text { - Segmented doses (low vs high) } \\
\text { - Time interval between the two drugs. }\end{array}$ & $\begin{array}{l}\text { - Di- or tri-iodine-131 labeled Hyp } \\
\text { - Replace iodine-131 with other beta or } \\
\text { alpha emitters, e.g., yttrium-90, actinium- } \\
\text { 225, lutetium-177, etc. }\end{array}$ \\
\hline
\end{tabular}

For targeted treatment of malignancies, so far a single dose of SMSDTTS has not brought radical treatment to R1 or RIF-1 tumors in rat and mouse models. The following reasons might account for such outcomes: a) tumors of the same origin in different organs or even in the same organ may respond differently to the same anticancer agent and inhomogeneity of vessel shutdown induced by CA4P must be taken into account for the SMSDTTS (104, Fig. 5); b) rapid repopulation of remaining tumor cells after CA4P; c) radiation induced cell death is relatively slow and less radical compared to the rapid tumor regrowth; d) physical decay of radioiodine-131 inside necrosis; and e) possible radioresistance following initial iodine-131 mediated locoreginal radiotherapy (93).
To further optimize and improve the anticancer efficacy with SMSDTTS, several approaches are considered to be implemented (Table 1). For instance, anti-angiogenesis agents may be employed to provide synergistic effects for preventing tumor regrowth (105); consecutive doses of ${ }^{131}$ I-Hyp after one CA4P treatment, or multi-cycle treatments of SMSDTTS might be better options for malignancies. Other radionuclides with longer half-life and/or higher potency of radiation, and deeper penetration range in soft tissue, are supposed to be substitutional for iodine-131. Recently, radiosyntheses of di- and tri-131I radioiodinated-Hyp are proposed to double and triple the radio-therapeutic efficacy relative to the current mono-131I-Hyp. 


\section{From preclinical experiments towards possible clinical applications}

Translating laboratory research into approved clinical anticancer therapy is the major goal for preclinical research on various anticancer drugs or strategies. Laboratory results have assured the prominent in vivo and ex vivo necrosis targeting property of 131I-Hyp. Proofs of potency for detection of necrotic diseases and radio-targeting of malignancies using radioiodinated Hyp have been demonstrated in small animals with different types of tumors. Clinical trials using SMSDTTS in veterinary and human cancer patients have now been planned in order to evaluate the safety and efficacy of SMSDTTS in real clinical scenarios.

A variety of requirements must be fulfilled in order to bring appealing outcomes from laboratory bench towards novel therapy available for clinical patients with malignancies. To acquire first-in-man compelling proofs, SMSDTTS or each single agent involved must be proven better than the standard of care, which will be followed by confirmatory trials in cancer patients of various indications, prior to the potential final regulatory approval of SMSDTTS.

After being approved by the ethical committee and officially documented by certain clinical trial registry, phase 0 studies using micro-dosing of 123I-Hyp will be conducted in healthy volunteers and liver cancer patients having received radiofrequency ablation to investigate tracer biodistribution in normal organs and targetability in therapeutic necrosis. This will be followed by phase I study on dose finding and safety of the involved drugs, and further by phase II or more advanced studies on therapeutic efficacies. Hypervascular primary and metastatic solid malignancies after being treated and failed with existing therapies will be considered as indications for such clinical trials. Contrast enhanced MRI will be used to identify eligible tumors to be treated and to follow up the therapeutic outcomes. Both the tumor volume change and patient survival will be used as end-points for the evaluations on the clinical trials.

\section{Conclusion}

The desired diagnostic capacities, potential therapeutic efficacies, good in vivo tolerance, and low toxicity of radioiodinated Hyp have been evaluated in animal experiments. The SMSDTTS based on combined sequential systemic delivery of a VDA and iodine-131 labeled mono-iodohypericin has been elaborated to introduce an unconventional but general approach to wipe out residual cancer cells. SMSDTTS has shown potentiality to treat multifocal and multi- type malignant tumors. By destroying the microenvironment, the SMSDTTS may also conceptually and technologically provide a solution for CSCs. Upon optimizations, SMSDTTS as a natural, versatile and simple anticancer strategy with greater probability of tumor response can be expected for future clinical applications.

\section{Abbreviations}

SMSDTTS: Small Molecule Sequential Dual-Targeting Theragnostic Strategy; CA4P: combretastatin A4 phosphate; CSCs: cancer stem cells 131I-Hyp: 131I-labeled mono-iodohypericin; VDA: vascular disrupting agent; MTD: maximum tolerated dose; NACAs: necrosis and necrosis avid contrast agents; MRI: magnetic resonance imaging; Gd-DTPA: gadopentetic acid; Mn-TPP: Mn-tetraphenylporphyrin; Gd-MP: bis-Gd-DTPAmesoporphyrin; ECIII-60: bis-Gd-DTPA-pamoic acid; ECIV-7: bis-Gd-DTPA-benzylidene-bis (indole-2-acetic acid hydrazide); mAb: monoclonal antibody; PDT: photodynamic therapy; SPECT: single-photon emission computed tomography; R1: rhabdomyosarcoma-1; RIF-1: radiation-induced fibrosarcoma; I-Hyp: non-radioactive mono-iodohypericin; $\mathrm{LD}_{50}$ : half lethal dose; DMSO: dimethyl sulfoxide.

\section{Acknowledgments}

This work was partially supported by the grants awarded by FWO Vlaanderen ZWAP/05/018; Geconcerteerde Onderzoeksactie of the Flemish Government, OT project (OT/06/70); the KU Leuven Molecular Small Animal Imaging Center MoSAIC (KUL EF/05/08); the center of excellence In vivo Molecular Imaging Research (IMIR) of KU Leuven; the IWT SBO 'Imagine' (SBO80017), the National Natural Science Foundation of China (81071828), Jiangsu Province Natural Science Foundation (BK2010594) and a EU project Asia-Link CfP 2006-EuropeAid/ 123738/C/ACT/Multi-Proposal No. 128-498/111. The corresponding author Yicheng Ni is currently a Bayer Lecture Chair holder.

\section{Competing interests}

All the authors declare there is no any conflict of interest regarding the content of the paper. The authors have no financial relationship with any organizations that sponsored our research.

\section{References}

1. Praetorius NP, Mandal TK. Engineered nanoparticles in cancer therapy. Recent Pat Drug Deliv Formul. 2007; 1: 37-51. 
2. Aggarwal BB, Danda D, Gupta S, Gehlot P. Models for prevention and treatment of cancer: problems vs promises. Biochem Pharmacol. 2009; 78: 1083-94.

3. Li J, Chen F, Cona MM, Feng Y, Himmelreich U, Oyen R, et al. A Review on Various Targeted Anticancer Therapies. Targeted Oncol. 2012; 7: 69-85.

4. Fidler IJ. Cancer metastasis. Br Med Bull. 1991; 47: 157-77.

5. Liu K, Caldwell SA, Greeneltch KM, Yang D, Abrams SI. CTL adoptive immunotherapy concurrently mediates tumor regression and tumor escape. J Immunol. 2006; 176: 3374-82.

6. Geiger TR, Peeper DS. Metastasis mechanisms. Biochimica et Biophysica Acta (BBA). 2009; 1796: 293-308.

7. Bell LK, Ainsworth NL, Lee SH, Griffiths JR. MRI \& MRS assessment of the role of the tumour microenvironment in response to therapy. NMR Biomed. 2011; 24: 612-35.

8. Chen FM, Taylor CR, Epstein AL. Tumor necrosis treatment of ME-180 human cervical carcinoma model with 131I-labeled TNT-1 monoclonal antibody. Cancer Res. 1989; 49: 4578-85.

9. Khawli LA, Mizokami MM, Sharifi J, Hu P, Epstein AL. Pharmacokinetic characteristics and biodistribution of radioiodinated chimeric TNT-1, -2, and -3 monoclonal antibodies after chemical modification with biotin. Cancer Biother Radiopharm. 2002; 17: 359-70.

10. Shapiro WR, Carpenter SP, Roberts K, Shan JS. (131)I-chTNT-1/B mAb: tumour necrosis therapy for malignant astrocytic glioma. Expert Opin Biol Ther. 2006; 6: 539-45.

11. Li J, Sun Z, Zhang J, Shao H, Miranda Cona M, et al. A Dual-targeting Anticancer Approach: Soil and Seed Principle. Radiology. 2011; 260:799-807.

12. Hackshaw A, Harmer C, Mallick U, Haq M, Franklyn JA. ${ }^{131}$ I activity for remnant ablation in patients with differentiated thyroid cancer: A systematic review. J Clin Endocrinol Metab. 2007; 92:28-38.

13. Papadimitriou D, Kottou S, Oros L, Ilias I, Molfetas M, et al. Differentiated thyroid cancer: comparison of therapeutic iodine 131 biological elimination after discontinuation of levothyroxine versus administration of recombinant human thyrotropin. Annals of Nuclear Medicine. 2006; 20: 63-67.

14. Brown JM, Giaccia AJ. The unique physiology of solid tumors: opportunities (and problems) for cancer therapy. Cancer Res 1998; 58: 1408-16.

15. Ruoslahti E. Specialization of tumour vasculature. Nature Reviews Cancer. 2012; 2: 83-90.

16. Denekamp J, Hill S. Angiogenic attack as a therapeutic strategy for cancer. Radiother Oncol. 1991; 20: 103-12.

17. Cooney MM, van Heeckeren W, Bhakta S, Ortiz J, Remick SC. Drug insight: vascular disrupting agents and angiogenesis--novel approaches for drug delivery. Nat Clin Pract Oncol. 2006; 3: 682-92.

18. Schnitzer JE. Vascular targeting as a strategy for cancer therapy. N Engl J Med. 1998; 339: 472-4.

19. Arap W, Pasqualini R, Ruoslahti E. Cancer treatment by targeted drug delivery to tumor vasculature in a mouse model. Science. 1998; 279: 377-80.

20. Siemann DW, Horsman MR. Vascular targeted therapies in oncology. Cell Tissue Res. 2009; 335: 241-8.

21. Tozer GM, Kanthou C, Baguley BC. Disrupting tumour blood vessels. Nat Rev Cancer. 2005; 5: 423-35.

22. Cooney MM, Ortiz J, Bukowski RM, Remick SC. Novel vascular targeting/disrupting agents: combretastatin A4 phosphate and related compounds. Curr Oncol Rep. 2005; 7: 90-5.

23. Ching LM, Zwain S, Baguley BC. Relationship between tumour endothelial cell apoptosis and tumour blood flow shutdown following treatment with the antivascular agent DMXAA in mice. Br J Cancer. 2004; 90: 906-10.

24. Seshadri M, Spernyak JA, Maiery PG, Cheney RT, Mazurchuk R, et al. Visualizing the acute effects of vascular-targeted therapy in vivo using intravital microscopy and magnetic resonance imaging: correlation with endothelial apoptosis, cytokine induction, and treatment outcome. Neoplasia. 2007; 9:128-35.

25. McPhail LD, McIntyre DJ, Ludwig C, Kestell P, Griffiths JR, et al. Rat tumor response to the vascular-disrupting agent 5,6-dimethylxanthenone-4-acetic acid as measured by dynamic contrast-enhanced magnetic resonance imaging, plasma 5-hydroxyindoleacetic acid levels, and tumor necrosis. Neoplasia. 2006; 8: 199-206.

26. Ching LM, Goldsmith D, Joseph WR, Körner H, Sedgwick JD, et al. Induction of intratumoral tumor necrosis factor (TNF) synthesis and hemorrhagic necrosis by 5,6-dimethylxanthenone-4-acetic acid (DMXAA) in TNF knockout mice. Cancer Res. 1999; 59: 3304-7.
27. Wang H, Cona MM, Li J, Feng Y, Chen F, et al. The Role of Modern Medical Imaging in Evaluation of Tumor Response to Vascular Targeting Therapy. Recent Researches in Modern Medicine. 2009; ISBN: 978-960-474-278-3.

28. Honore S, Pasquier E, Braguer D. Understanding microtubule dynamics for improved cancer therapy. Cell Mol Life Sci. 2005; 62: 3039-56.

29. Siemann DW. The unique characteristics of tumor vasculature and preclinical evidence for its selective disruption by Tumor-Vascular Disrupting Agents. Cancer Treat Rev. 2010; 37: 63-74.

30. Mason RP, Zhao D, Liu L, Trawick ML, Pinney KG. A perspective on vascular disrupting agents that interact with tubulin: preclinical tumor imaging and biological assessment. Integr Biol (Camb). 2011; 3: 375-87.

31. Tozer GM, Kanthou C, Lewis G, Prise VE, Vojnovic B, et al. Tumour vascular disrupting agents: combating treatment resistance. $\mathrm{Br} J$ Radiol. 2008; 81: S12-20.

32. Dome B, Hendrix MJ, Paku S, Tovari J, Timar J. Alternative vascularization mechanisms in cancer: Pathology and therapeutic implications. Am J Pathol.2007; 170: 1-15.

33. Ma WW, Adjei AA. Novel agents on the horizon for cancer therapy. CA Cancer J Clin. 2009; 59: 111-37.

34. Zweifel M, Jayson GC, Reed NS, Osborne R, Hassan B, et al. Phase II trial of combretastatin A4 phosphate, carboplatin, and paclitaxel in patients with platinum-resistant ovarian cancer. Ann Oncol. 2011; 22: 2036-41.

35. Rustin GJ, Galbraith SM, Anderson H, Stratford M, Folkes LK, et al. Phase I clinical trial of weekly combretastatin A4 phosphate: clinical and pharmacokinetic results. J Clin Oncol. 2003; 21: 2815-22.

36. Zweifel M, Rustin G. The clinical development of tubulin binding vascular disrupting agents. Vascular disruptive agents for the treatment of cancer 2010;: 183-216.

37. Tozer GM, Prise VE, Wilson J, Cemazar M, Shan S, et al. Mechanisms associated with tumor vascular shut-down induced by combretastatin A-4 phosphate: intravital microscopy and measurement of vascular permeability. Cancer Res. 2001; 61: 6413-22.

38. Tozer GM, Prise VE, Lewis G, Xie S, Wilson I, et al. Nitric oxide synthase inhibition enhances the tumor vascular-damaging effects of combretastatin a-4 3-o-phosphate at clinically relevant doses. Clin Cancer Res. 2009; 15: 3781-90.

39. Salmon BA, Siemann DW. Characterizing the tumor response to treatment with combretastatin A4 phosphate. Int J Radiat Oncol Biol Phys. 2007; 68: 211-7.

40. Rustin GJ, Shreeves G, Nathan PD, Gaya A, Ganesan TS, et al. A Phase Ib trial of CA4P (combretastatin A-4 phosphate), carboplatin, and paclitaxel in patients with advanced cancer. Br J Cancer. 2010; 102: 1355-60.

41. Mitrus I, Sochanik A, Cichon T, Szala S. Combination of combretastatin A4 phosphate and doxorubicin-containing liposomes affects growth of B16-F10 tumors. Acta Biochim Pol. 2009; 56: 161-5.

42. Bilenker JH, Flaherty KT, Rosen M, Davis L, Gallagher M, et al. Phase I trial of combretastatin a-4 phosphate with carboplatin. Clin Cancer Res. 2005; 11: 1527-33.

43. Degterev A, Yuan J. Expansion and evolution of cell death programmes. Nat Rev Mol Cell Biol. 2008; 9: 378-90.

44. Korostoff J, Wang JF, Kieba I, Miller M, Shenker BJ, et al. Actinobacillus actinomycetemcomitans leukotoxin induces apoptosis in HL-60 cells. Infect Immun. 1998; 66: 4474-83.

45. Dasari M, Lee S, Sy J, Kim D, Brown M, et al. Hoechst-IR: an imaging agent that detects necrotic tissue in vivo by binding extracellular DNA. Org Lett. 2010; 12: 3300-3.

46. Walker NI, Harmon BV, Gobe GC, Kerr JF. Patterns of cell death. Methods Achiev Exp Pathol. 1988; 13: 18-54.

47. Ni Y, Bormans G, Chen F, Verbruggen A, Marchal G. Necrosis avid contrast agents: functional similarity versus structural diversity. Invest Radiol. 2005; 40: 526-35.

48. Ni Y, Marchal G, Yu J, Lukito G, Petre C, et al. Localization of metalloporphyrin-induced "specific" enhancement in experimental liver tumors: comparison of magnetic resonance imaging, microangiographic, and histologic findings. Acad Radiol.1995; 2: 687-99.

49. Ni Y, Marchal G, van Damme B, van Hecke P, Michiels J, et al. Magnetic resonance imaging, microangiography, and histology in a rat model of primary liver cancer. Invest Radiol. 1992; 27: 689-97.

50. Flotats A, Carrio I. Non-invasive in vivo imaging of myocardial apoptosis and necrosis. Eur J Nucl Med Mol Imaging. 2003; 30: 615-30.

51. Ni Y, Petre C, Miao Y, Yu J, Cresens E, et al. Magnetic resonance imaging-histomorphologic correlation studies on paramagnetic metalloporphyrins in rat models of necrosis. Invest Radiol. 1997; 32: 770-779.

52. Ni Y, Adzamli K, Miao Y, Cresens E, Yu J, et al. MRI contrast enhancement of necrosis by MP-2269 and gadophrin-2 in a rat model of liver infarction. Invest Radiol. 2001; 36: 97-103. 
53. Ni Y. Metalloporphyrins and Functional Analogues as MRI Contrast Agents. Current Medical Imaging Reviews. 2008; 4: 96-112.

54. Oostendorp M, Post MJ, Backes WH. Vessel growth and function: depiction with contrast-enhanced MR imaging. Radiology. 2009; 251: 317-35.

55. Weissleder R, Lee A, Khaw B, Shen T, Brady T. Antimyosin-labeled monocrystalline iron oxide allows detection of myocardial infarct: MR antibody imaging. Radiology.1992; 182: 381-5.

56. Holman BL, Chisholm RJ, Braunwald E. The prognostic implications of acute myocardial infarct scintigraphy with 99mTc-pyrophosphate. Circulation.1978; 57: 320-6

57. Kaye M, Silverton S, Rosenthall L. Technetium-99m-pyrophosphate: studies in vivo and in vitro. J Nucl Med.1975; 16: 40-5.

58. Ni Y, Huyghe D, Verbeke K, de Witte PA, Nuyts J, et al. First preclinical evaluation of mono-[123I]iodohypericin as a necrosis-avid tracer agent. Eur J Nucl Med Mol Imaging. 2006; 33: 595-601.

59. De Saint-Hubert M, Prinsen K, Mortelmans L, Verbruggen A, Mottaghy F. Molecular imaging of cell death. Methods. 2009; 48: 178-87.

60. Karioti A, Bilia AR. Hypericins as Potential Leads for New Therapeutics. Int J Mol Sci. 2010; 11: 562-594.

61. Chen B, Zupko I, de Witte PA. Photodynamic therapy with hypericin in a mouse P388 tumor model: vascular effects determine the efficacy. Int J Oncol. 2001; 18: 737-42.

62. Chen B, Roskams T, de Witte PA. Antivascular tumor eradication by hypericin-mediated photodynamic therapy. Photochem Photobiol. 2002; 76: 509-13.

63. Agostinis P, Vantieghem A, Merlevede W, de Witte PA. Hypericin in cancer treatment: more light on the way. Int J Biochem Cell Biol. 2002; 34 : 221-41.

64. Sattler S, Schaefer U, Schneider W. Hoelzl J, Lehr CM. Binding, uptake, and transport of hypericin by Caco-2 cell monolayers. J Pharm Sci.1997; 86: 1120-6.

65. Thomas C, Pardini RS. Oxygen dependence of hypericin-induced phototoxicity to EMT6 mouse mammary carcinoma cells. Photochem Photobiol. 1992; 55: 831-7.

66. Vandenbogaerde AL, Cuveele JF, Proot P, Himpens BE, Merlevede WJ, et al. Differential cytotoxic effects induced after photosensitization by hypericin. J Photochem Photobiol B. 1997; 38: 136-42.

67. English DS, Doyle RT, Petrich JW, Haydon PG. Subcellular distributions and excited-state processes of hypericin in neurons. Photochem Photobiol.1999; 69: 301-5.

68. Ni Y, Bormans G, Marchal G, Verbruggen A. Necrosis avid tracer agent. European Patent, Belgium. 2003. Patent Application Ref No. LRD-GB-1-455.

69. Fonge H, Van de Putte M, Huyghe D, Bormans G, Ni Y, et al. Evaluation of tumor affinity of mono-[(123)I]iodohypericin and mono-[(123)I]iodoprotohypericin in a mouse model with a RIF-1 tumor. Contrast Media Mol Imaging.2007; 2: 113-9.

70. Fonge H, Vunckx K, Wang H, Feng Y, Mortelmans L, et al. Non-invasive detection and quantification of acute myocardial infarction in rabbits using mono-[123I]iodohypericin microSPECT. Eur Heart J. 2008; 29: 260-9.

71. Van de Putte M, Ni Y, De Witte PA. Exploration of the mechanism underlying the tumor necrosis avidity of hypericin. Oncol Rep. 2008; 19: 921-6.

72. Van de Putte M, Wang H, Chen F, De Witte PA, Ni Y. Hypericin as a marker for determination of tissue viability after radiofrequency ablation in a murine liver tumor model. Oncol Rep. 2008; 19: 927-32.

73. Van de Putte M, Wang H, Chen F, de Witte PA, Ni Y. Hypericin as a marker for determination of tissue viability after intratumoral ethanol injection in a murine liver tumor model. Acad Radiol. 2008; 15: 107-13.

74. Carlsson J, Forssell-Aronsson E, Glimelius B, Mattsson S. Therapy with radiopharmaceuticals. Acta Oncol.2002; 41: 623-8.

75. Epstein AL, Chen FM, Taylor CR. A novel method for the detection of necrotic lesions in human cancers. Cancer Res.1988; 48: 5842-8.

76. Cona MM, Wang H, Li J, Feng Y, Chen F, et al. Continuing pursuit for ideal systemic anticancer radiotherapeutics. Invest New Drugs. 2012; 30:2050-65.

77. Goldenberg DM, Griffiths GL. Radioimmunotherapy of cancer: arming the missiles. J Nucl Med. 1992; 33: 1110-2.

78. Stern M, Herrmann R. Overview of monoclonal antibodies in cancer therapy: present and promise. Crit Rev Oncol Hematol. 2005; 54: 11-29.

79. Van de Putte M, Marysael T, Fonge H, Roskams T, Cona MM, et al. Radiolabeled iodohypericin as tumor necrosis avid tracer: diagnostic and therapeutic potential. Int J Cancer. 2011; 131: 129-137

80. Ni Y, Pislaru C, Bosmans H, Pislaru S, Miao Y, et al. Intracoronary delivery of Gd-DTPA and Gadophrin-2 for determination of myocardial viability with MR imaging. Eur Radiol. 2001; 11: 876-83.
81. Ginj M, Zhang H, Waser B, Cescato R, Wild D, et al. Radiolabeled somatostatin receptor antagonists are preferable to agonists for in vivo peptide receptor targeting of tumors. Proc Natl Acad Sci U S A. 2006; 103: $16436-41$.

82. Li J, Cona MM, Chen F, Zhou L, Nuyts J, et al. Systemic Sequential Administrations of Combretastatin A4 Phosphate and Radioiodinated Hypericin: Synergistic Targeted Anticancer Effect with Prolonged Survival in a Murine Model of Subcutaneous Tumor Xenografts. Theranostics. (In press).

83. Hu CM, Aryal S, Zhang L. Nanoparticle-assisted combination therapies for effective cancer treatment. Therapeutic Delivery. 2010; 1: 323-334.

84. Maione P, Gridelli C, Troiani T, Ciardiello F. Combining targeted therapies and drugs with multiple targets in the treatment of NSCLC. Oncologist. 2006; 11: 274-84.

85. Wang H, Marchal G, Ni Y. Multiparametric MRI biomarkers for measuring vascular disrupting effect on cancer. World J Radiol. 2011; 3: 1-16.

86. Nagaiah G, Remick SC. Combretastatin A4 phosphate: a novel vascular disrupting agent. Future Oncol. 2010; 6: 1219-28.

87. Siemann DW, Shi W. Dual targeting of tumor vasculature: combining Avastin and vascular disrupting agents (CA4P or OXi4503). Anticancer Res. 2008; 28: 2027-31.

88. Ng QS, Mandeville H, Goh V, Alonzi R, Milner J, et al. Phase Ib trial of radiotherapy in combination with combretastatin-A4-phosphate in patients with non-small-cell lung cancer, prostate adenocarcinoma, and squamous cell carcinoma of the head and neck. Ann Oncol. 2005; 23: 231-7.

89. Marysael T, Ni Y, Lerut E, de Witte P. Influence of the vascular damaging agents DMXAA and ZD6126 on hypericin distribution and accumulation in RIF-1 tumors. J Cancer Res Clin Oncol. 2011;137:1619-27.

90. Li J, Cona MM, Chen F, Zhou L, Nuyts J, et al. Exploring Diagnostic and Therapeutic Potentials of Radioiodinated Hypericin in Animal Models of Pathologies. Theranostics. 2012; 2: 1010-1019.

91. Jain RK. Normalization of tumor vasculature: an emerging concept in antiangiogenic therapy. Science. 2005; 307: 58-62.

92. Verbruggen A, Coenen $\mathrm{HH}$, Deverre JR, Guilloteau D, Langstrom B, Salvadori PA, Halldin C. Guideline to regulations for radiopharmaceuticals in early phase clinical trials in the EU. Eur J Nucl Med Mol Imaging. 2008; 35:2144-215.

93. Misaki T, Iwata M, Iida Y, Kasagi K, Konishi J. Chemo-radionuclide therapy for thyroid cancer: Initial experimental study with cultured cells. Annals of Nuclear Medicine. 2002; 16: 403-408

94. Cona MM, Li J, Chen F, Feng Y, Alpizar Y, et al. A safety study on single intravenous dose of tetrachloro-diphenyl glycoluril [Iodogen] dissolved in dimethyl sulfoxide [DMSO]. XENOBIOTICA 2013; epub.

95. Li J. Cona MM, Feng Y, Chen F, Fu X, et al. A Single-dose toxicity study in mice on non-radioactive iodinated hypericin for a targeted anticancer therapy. Acta Pharmacologica Sinica. 2012; 33: 1549-1556.

96. Fonge $\mathrm{H}$, Jin L, Wang $\mathrm{H}$, Ni Y, Bormans $\mathrm{G}$, et al. Synthesis and preliminary evaluation of mono-[123I]iodohypericin monocarboxylic acid as a necrosis avid imaging agent. Bioorg Med Chem Lett. 2007; 17: 4001-5.

97. Bormans G, Huyghe D, Christiaen A, Verbeke K, de Groot T, et al. Preparation, analysis and biodistribution in mice of iodine-123 labelled derivatives of hypericin. J Label Compd Radiopharm. 2004; 47: 191-198.

98. Belhocine TZ, Prato FS. Transbilayer phospholipids molecular imaging. EJNMMI Res. 2011; 1: 17.

99. Watanabe N, Yokoyama K, Kinuya S, Shuke N, Shimizu M, et al. Radiotoxicity after iodine-131 therapy for thyroid cancer using the micronucleus assay. J Nucl Med.1998; 39: 436-40.

100. Shapiro B, Sisson JC, Wieland DM, Mangner TJ, Zempel SM, et al. Radiopharmaceutical therapy of malignant pheochromocytoma with [131I]metaiodobenzylguanidine: results from ten years of experience. J Nucl Biol Med.1991; 35: 269-76.

101. Narra VR, Howell RW, Harapanhalli RS, Sastry KS, Rao DV. Radiotoxicity of some iodine-123, iodine-125 and iodine-131-labeled compounds in mouse testes: implications for radiopharmaceutical design. J Nucl Med. 1992; 33: 2196-201.

102. Dowlati A, Robertson K, Cooney M et al. A phase I pharmacokinetic and translational study of the novel vascular targeting agent combretastatin a-4 phosphate on a single-dose intravenous schedule in patients with advanced cancer. Cancer Res. 2002; 62: 3408-3416.

103. Cooney MM, Radivoyevitch T, Dowlati A, et al. Cardiovascular safety profile of combretastatin a4 phosphate in a single-dose phase I study in patients with advanced cancer. Clin Cancer Res. 2004; 10: 96-100.

104. Li J, Chen F, Feng Y, Miranda Cona M, Yu J, et al. Diverse Responses to Vascular Disrupting Agent Combretastatin A4 Phosphate: A Comparative Study in Rats with Hepatic and Subcutaneous Tumor Allografts 
Using MRI Biomarkers, Microangiography and Histopathology. Translational Oncology. DOI: 10.1593/tlo.12367.

105. Chen F, Feng Y, Zheng K, De Keyzer F, Li J, et al. Enhanced Antitumor Efficacy of a Vascular Disrupting Agent Combined with an Antiangiogenic in a Rat Liver Tumor Model Evaluated by Multiparametric MRI. PLoS ONE. 2012; 7: e41140. 